\title{
The road to nearly zero energy buildings. Case of study: Spain. Comparison between CTE HE 2006 and 2013 in a simulated model.
}

\author{
D. Carmona ${ }^{(1)}$, D. De La Maya ${ }^{(2)}$, A. García ${ }^{(3)}$, L.A. Horrillo ${ }^{(4)}$ A.M. Reyes ${ }^{(5)}$ \\ (1)(2)(4)(5) Escuela de II.II. Avda. de Elvas, s/n. 06006-Badajoz. \\ (3) Facultad de Ciencias Económicas y Empresariales. Avda. de Elvas, s/n. 06006-Badajoz. \\ (1)924289600-Ext.86788. dcarmona@unex.es
}

\begin{abstract}
.
Achieving Nearly Zero-Energy Buildings (NZEBs) is a main goal for the European Union, in order to reduce energy consumption in the building sector. NZEB means a building that has a very high energy performance. Its energy requirements should be covered by renewable sources, produced on-site or nearby [1]. It could be possible if building were turned into a "small power generating station", or reducing consumption with passive building proposals. However, we think that it is worth looking for a balance between energy consumption and generation for every building, following this simple equation:
\end{abstract}

$$
\text { Consumption }=\text { demand }- \text { generation }
$$

The European regulations have already begun to indicate deadlines to implement NZEB requirements in buildings. Therefore, Spanish legislation related to energy efficiency and renewable energy generation in buildings has been recently updated, CTE HE [2].

This paper provides a comparative analysis for the new requirements (2013 CTE DB HE compared with previous 2006 regulation, revised in 2009).

This study was performed by using a computer building model, including its geometry, building materials, usage profiles and installations. Thus, we could compare the characteristics of the different regulations, and we could evaluate the progress toward the NZEB concept.

\section{Key words}

Power. Efficiency. Saving. Computer simulation. NZEB buildings.

\section{Introduction}

The study is based on the analysis of the energy saving regulation. We have worked with a computer model showing the differences between the requirements and verification methods included in the 2006 version (2009) and 2013 version of the "Documento de Ahorro de Energía del Código Técnico" (a public Spanish official document related with energy saving rules in building sector). We have also evaluated the progress towards the NZEB concept.

A Nearly Zero-Energy Building (NZEB) is the one that was conceive on the basis of energy efficiency systems, including severe regulatory requirements, and minimizing its energy supplies, which are covered to a very significant extent by energy from renewable sources (self-generating or imported from nearby areas).

Consumo energético de un edificio

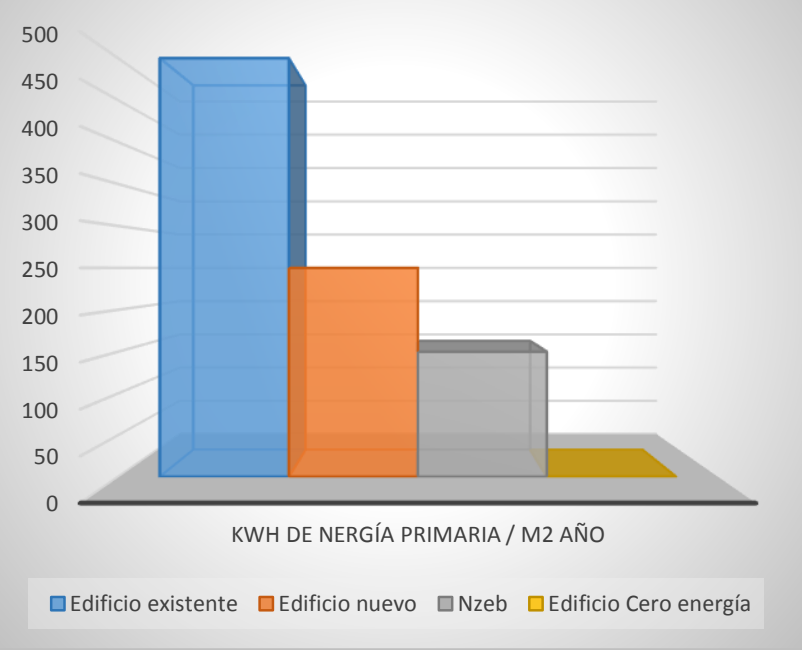

Fig. 1. Energy consumption at different kind of buildings. 


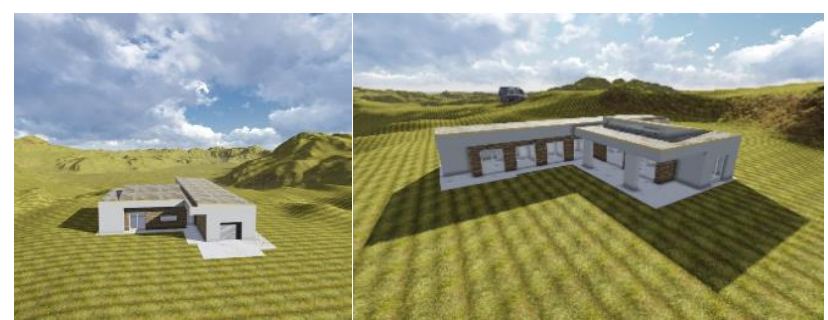

Fig. 2. Computer model used to perform the simulation.

In order to perform the comparison and to evaluate the differences, we have developed an example with a suposed new detached house, which observes the 2006 (2009) HE requirements. Then, the model simulates the 2013 HE requirements, including the corrections related with the building envelope and facilities to comply with directives.

\section{Experimental}

The building model consists of a single family house with four bedrooms, two bathrooms, kitchen, living room, sitting room, and garage, located at Badajoz (Spain).

\section{A. The simulation procedure.}

The procedure followed to make the comparative analysis of the CTE has been:

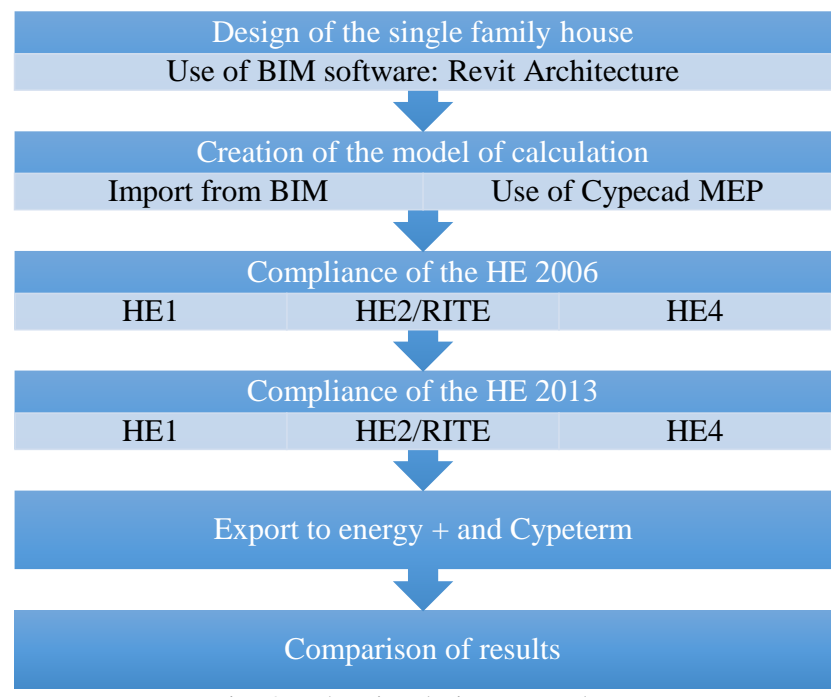

Fig. 3. The simulation procedure.

\section{B. Normative analysis of the new CTE DB HE 2013}

The energy consumption in Spain. According to the explanatory documentation from the CTE HE 2013 [2]:

"The building sector has a relevant impact both on global energy consumption in the country (only the residential sector represents $17 \%$ of the total final consumption) as in effect gas emissions greenhouse (more than one ton per household)." All this is part of a current energy context that our country is characterized by a high dependency from the outside, close to $80 \%$ and well above the European average of 54\%, resources which are limited and a future scenario of elevated energy prices".
In this context appear to European standards:

- Establish minimum requirements for energy efficiency in buildings.

- $\quad$ Requiring that new buildings built by 2020 (2018 in public buildings) are NZEB

This roadmap begins to apply in Spain through the CTE 2013 HE. It will allow reducing the traditional Spanish energy dependence, reducing the greenhouse gases (GHG) emissions, and increasing the building sector competitiveness.

It tries to get buildings with increasing comfort degrees for the user, as well as try to reduce the energy consumption.

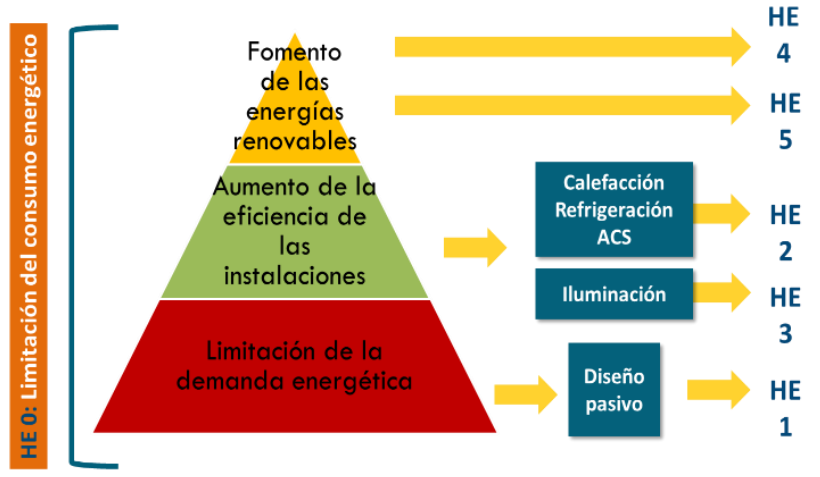

Fig. 4. 2013 HE description.

\section{Computer energy simulations.}

Using the fundamentals of the Energy Management Systems, and some architectural, structural recommendations, and from facilities, we have implemented:

The " integrated process design" (IPD). The IPD is defined by the International Energy Agency (IEA) as a procedure for building optimization, recognizing it as a comprehensive system across the life-cycle. It is based on interdisciplinary collaboration from the beginning until the end of the process.

If we model a building in a digital system and, then, we perform energy analysis, it is possible to determine:
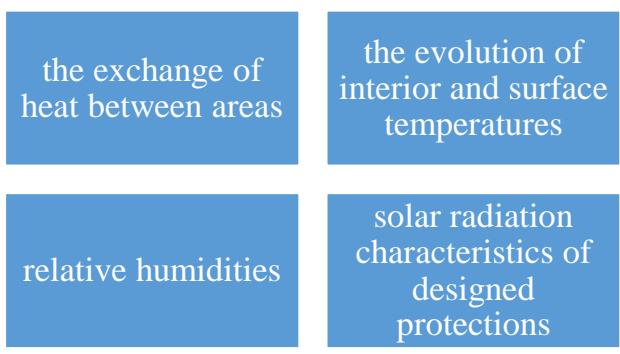

solar radiation

characteristics of

designed

protections

the energy demand (consumption)

according to thermal characteristics of enclosures 


\section{Results}

Next, the model simulation results are analyzed. In order to compare between different standards, we have consider the demand for thermal energy (thermal envelope study. HE1), the air conditioning installation (HE 2. RITE), and the solar thermal installation (HE 4).

\section{Thermal envelope study.}

It is worth noting that that, for the 2013 legal requirements, the level of isolation in the building envelope must be increased considerably.

\begin{tabular}{|c|c|c|}
\hline $\begin{array}{l}\text { Fachada rev } \\
\text { de hoja } \\
\text { autoportante }\end{array}$ & $\begin{array}{l}\text { con mortero monocapa, } \\
\text { ibrica, con trasdosado }\end{array}$ & $\begin{array}{r}\text { Superficie } \\
\text { total } \\
119.06 \mathrm{~m}^{2}\end{array}$ \\
\hline 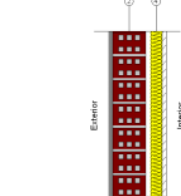 & $\begin{array}{l}\text { Listado de capas: } \\
1 \text { - Mortero monocapa } \\
2 \text { - Fábrica de ladrillo cerámico hueco } \\
3 \text { - Separación } \\
4 \text { - Lana de roca Confortpan } 208 \text { Roxul "ROCK } \\
5 \text { - Placa de yeso laminado } \\
6 \text { - Pintura plástica } \\
\text { Espesor total: }\end{array}$ & $\begin{array}{r}1.5 \mathrm{~cm} \\
11.5 \mathrm{~cm} \\
1.8 \mathrm{~cm} \\
4 \mathrm{~cm} \\
1.5 \mathrm{~cm} \\
--- \\
20.3 \mathrm{~cm}\end{array}$ \\
\hline
\end{tabular}

Limitación de demanda energética $\mathrm{U}_{\mathrm{m}}: 0.58 \mathrm{~W} /\left(\mathrm{m}^{2} \cdot \mathrm{K}\right)$

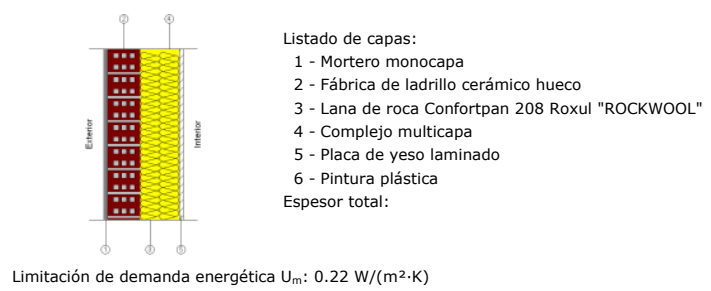

$1.5 \mathrm{~cm}$ $11.5 \mathrm{~cm}$ $6.8 \mathrm{~cm}$ $1.5 \mathrm{~cm}$ $28.1 \mathrm{~cm}$

Fig. 5. Building wall.

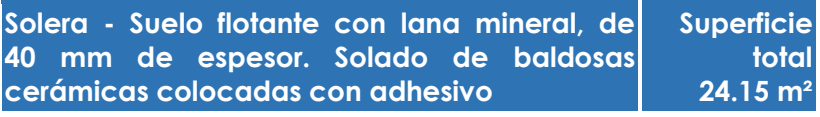

The decreasing in transmittance in the elements described in the tables above can be observed in the following graphs:

\section{Fachada}

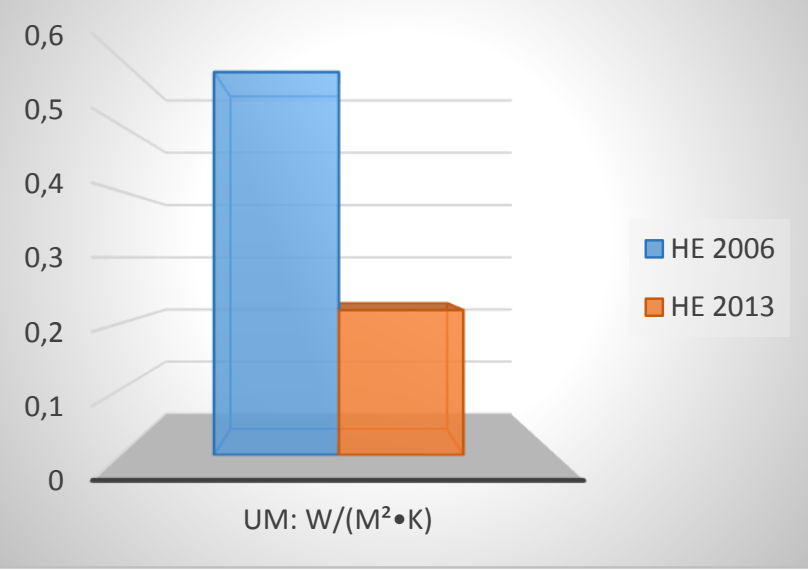

Fig. 7. Transmittance comparison (facade).

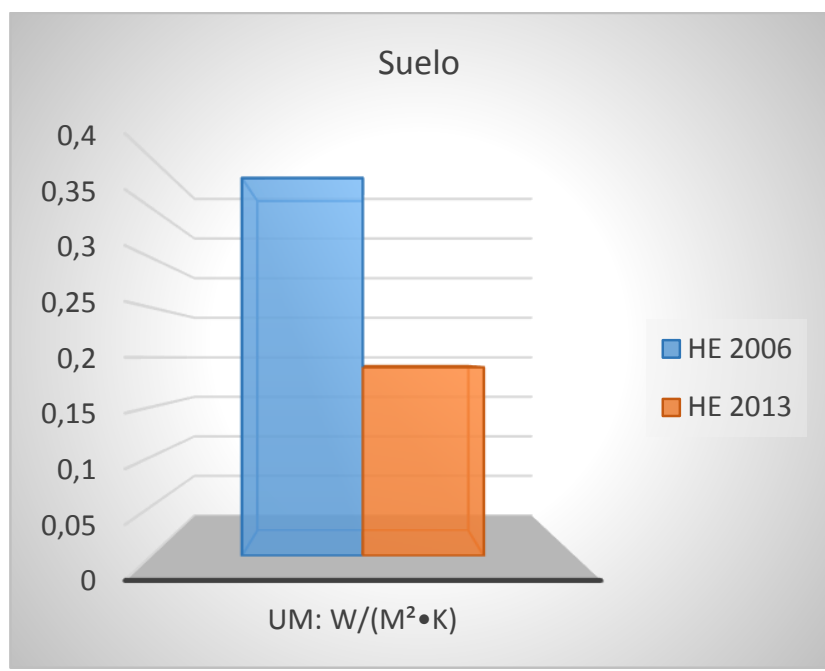

Fig. 8. Transmittance comparison (floor).

Building energy balance of the building (monthly).

- Solado de baldosas cerámicas de gres esmaltado $\quad 1 \mathrm{~cm}$ Mortero autonivelante de cemento $\quad 4 \mathrm{~cm}$ 4 - Lana mineral

5 - Solera de hormigón en masa

Espesor total:

Accounting for energy lost or gained by thermal transmission outside through heavy and light elements (Qtr, op and Qtr, w, respectively), the energy involved in the thermal link between areas (Qtr, ac), the energy exchanged by ventilation (Qve) gain net sensitive internal (Qint, s), the net solar gain (Qsol), the heat transferred or stored in the thermal mass of the building (Qedif), and the necessary contribution of $(\mathrm{QH})$ heating and cooling (QC).

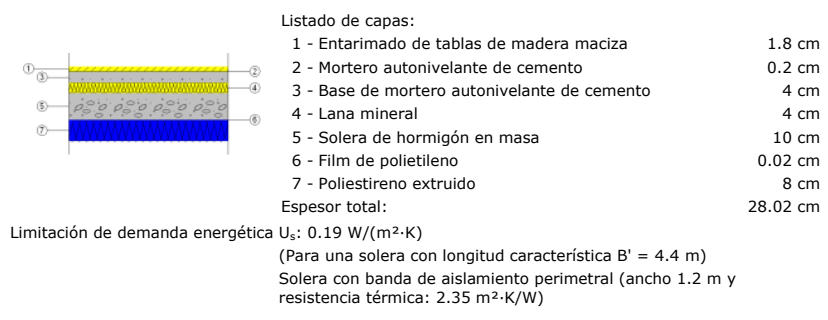

Fig. 6. Building floor.

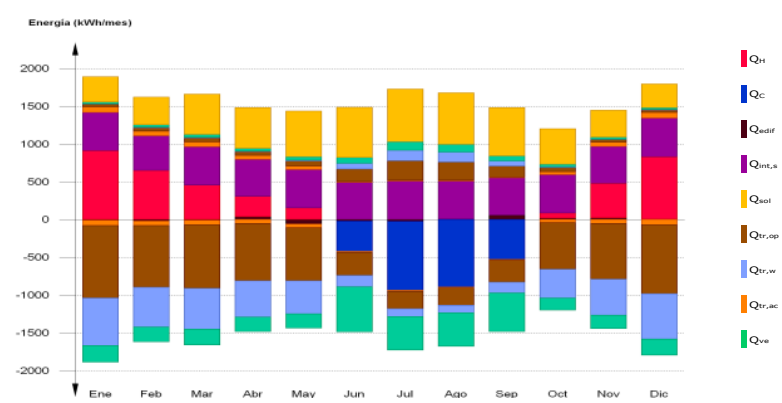

Fig. 9. Monthly energy balance. 
HE1 requirements: heating.

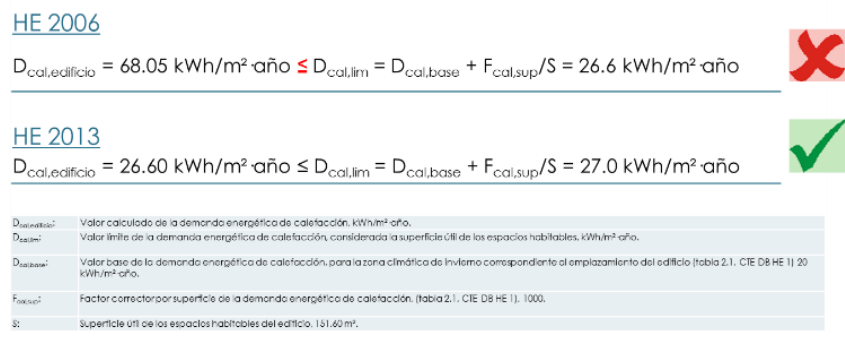

Fig. 10. HE1 requirements: heating.

HE1 requirements: cooling.

HE 2006

$D_{\text {ref,edificio }}=27.53 \mathrm{kWh} / \mathrm{m}^{2} \cdot$ año $\leq D_{\text {ref,lim }}=20.0 \mathrm{kWh} / \mathrm{m}^{2} \cdot$ año

HE 2013

$\underline{D_{\text {ref.edificio }}}=19.15 \mathrm{kWh} / \mathrm{m}^{2} \cdot a \tilde{n} o \leq D_{\text {ref.lim }}=20.0 \mathrm{kWh} / \mathrm{m}^{2} \cdot$ año

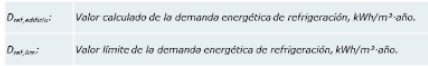

Fig. 11. HE1 requirements: cooling.

Energy demand for heating and cooling (monthly):
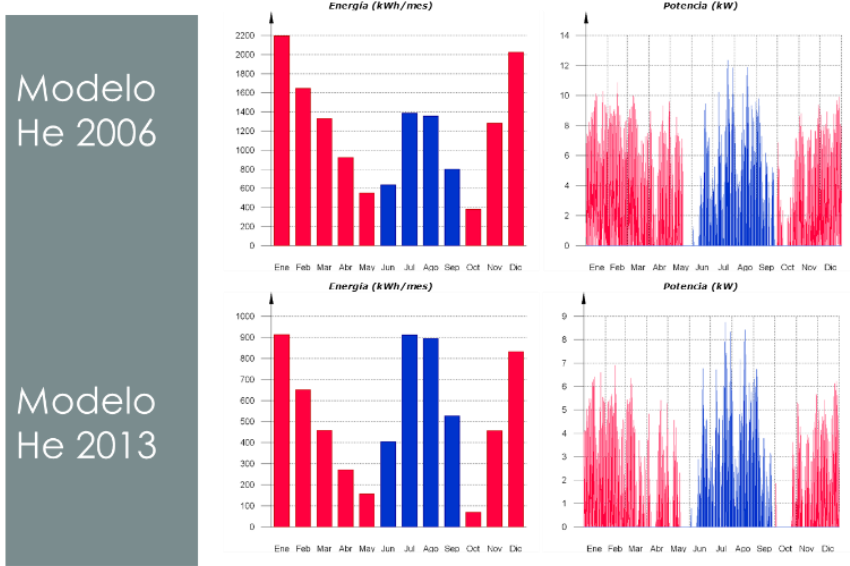

Fig. 12. Monthly energy demand.

Superimposed daily demand:
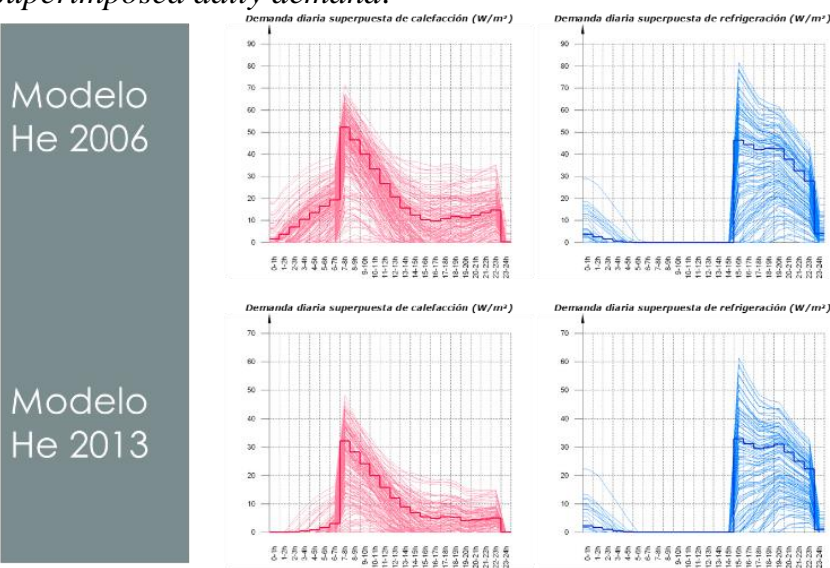

Fig. 13. Superimposed daily demand.
Typical demand by active day in model:

\section{Modelo He 2006}

\begin{tabular}{|c|c|c|c|c|c|c|}
\hline & $N^{\circ}$ activ. & $\begin{array}{l}\text { No días activos } \\
\text { (d) }\end{array}$ & $\begin{array}{c}N^{0} \text { horas } \\
\text { activas } \\
\text { (h) }\end{array}$ & $\begin{array}{l}\text { No horas por } \\
\text { activ. } \\
\text { (h) }\end{array}$ & $\begin{array}{l}\text { Potencia típica } \\
\left(\mathrm{W} / \mathrm{m}^{2}\right)\end{array}$ & $\begin{array}{l}\text { Demanda } \\
\text { típica por día } \\
\text { activo } \\
\left(\mathrm{kWh} / \mathrm{m}^{2}\right)\end{array}$ \\
\hline Calefacción & 264 & 223 & 3452 & 15 & 19.71 & 0.3051 \\
\hline Refrigeración & 110 & 110 & 948 & 8 & 29.04 & 0.2503 \\
\hline
\end{tabular}

\section{Modelo He 2013}

\begin{tabular}{|c|c|c|c|c|c|c|}
\hline & No activ. & $\begin{array}{l}\text { Ne días activos } \\
\text { (d) }\end{array}$ & $\begin{array}{l}\text { No horas } \\
\text { activas } \\
\text { (h) }\end{array}$ & $\begin{array}{l}\text { No horas por } \\
\text { activ. } \\
\text { (h) }\end{array}$ & $\begin{array}{c}\text { Potencia } \\
\text { tipica } \\
\left(\mathrm{W} / \mathrm{m}^{2}\right)\end{array}$ & $\begin{array}{l}\text { Demanda } \\
\text { típica por día } \\
\text { activo } \\
\left(\mathrm{kWh} / \mathrm{m}^{2}\right)\end{array}$ \\
\hline Calefacción & 224 & 195 & 2226 & 11 & 11.95 & 0.1364 \\
\hline Refrigeración & 110 & 108 & 873 & 8 & 21.93 & 0.1773 \\
\hline
\end{tabular}

Fig. 14. Typical demand by active day.

Indoor temperature evolution in model areas.

Evolution of minimum, maximum and average temperatures each day, along with the daily mean outside temperature.

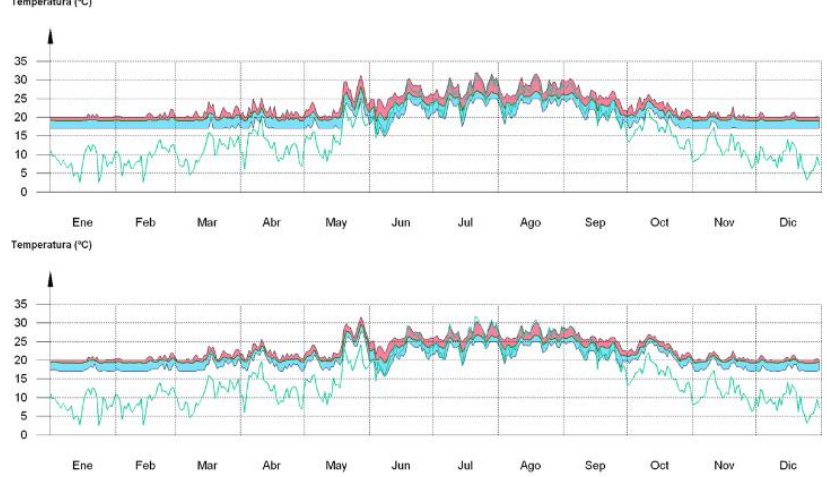

Fig. 15. Indoor temperature evolution.

Energy comparison (two cases).

The differences between demands of heating and cooling for the two studied cases can be observed in the following graphs:

\section{Calefacción}

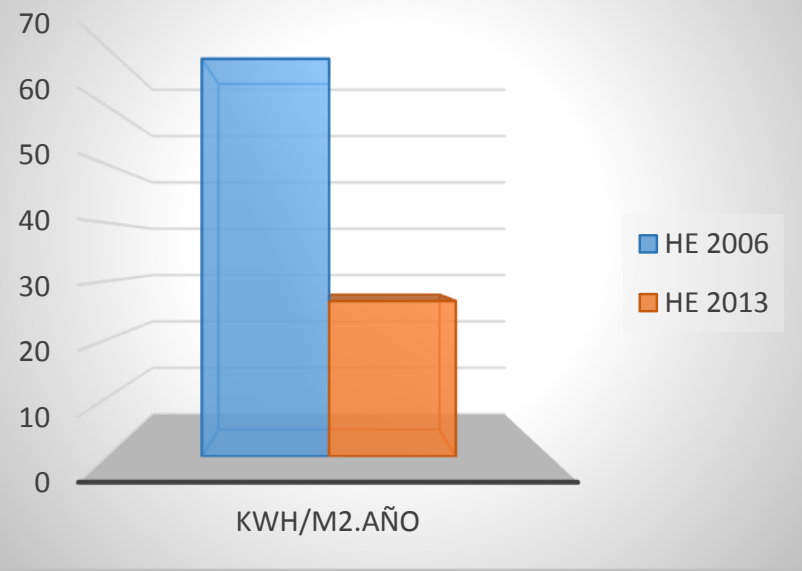

Fig. 16. Heating demand. 


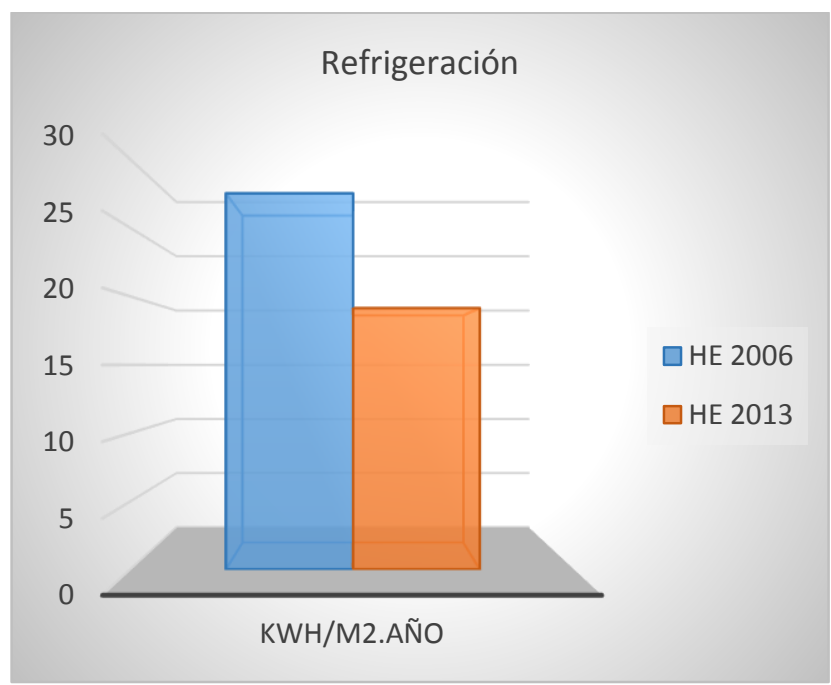

Fig. 17. Cooling demand.

Heating installation. CTE HE 2. RITE.

The decrease in thermal demand implies the possibility of reducing the power of the air conditioning installation, reducing the initial price of the equipment and its operation cost.

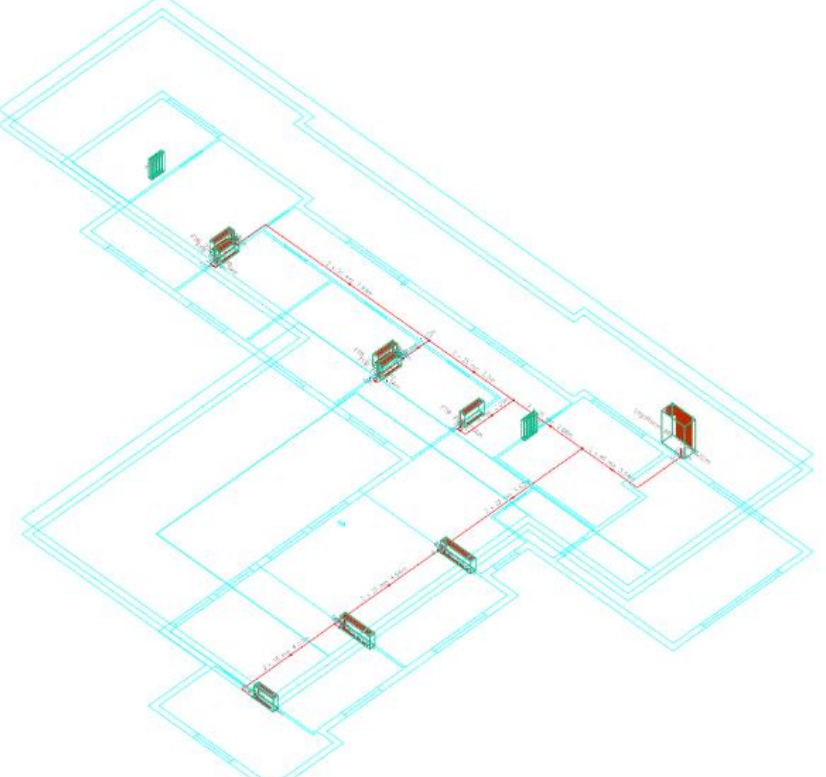

Fig. 18. Calculated air conditioning installation.

We can notice the savings if we analyze the next comparison between the needs of units not autonomous heating system (fan-coils) for the air conditioning of the housing:

\begin{tabular}{|c|c|c|c|c|c|}
\hline \multicolumn{6}{|c|}{ Fancoils } \\
\hline \multirow[t]{2}{*}{ Modelo } & & & & & \\
\hline & (W) & & $(1 / s)$ & $(\mathrm{kPa})$ & $(\mathrm{kPa})$ \\
\hline FTW 200 (A6-Planta baja) & 1400.0 & 2300.0 & 0.07 & 1.200 & 7.955 \\
\hline FTW 200 (A7-Planta baja) & 1400.0 & 2300.0 & 0.07 & 1.200 & 8.434 \\
\hline FTW 200 (A8-Planta baja) & 1400.0 & 2300.0 & 0.07 & 1.200 & 5.347 \\
\hline FTW 200 (A9-Planta baja) & 1400.0 & 2300.0 & 0.07 & 1.200 & 5.487 \\
\hline FTW 400 (A10-Planta baja) & 3530.0 & 4470.0 & 0.17 & 9.600 & 4.598 \\
\hline FTW 400 (A11-Planta baja) & 3530.0 & 4470.0 & 0.17 & 9.600 & 6.558 \\
\hline FTW 200 (A12-Planta baja) & 1400.0 & 2300.0 & 0.07 & 1.200 & 9.175 \\
\hline FTW 200 (A13-Planta baja) & 1400.0 & 2300.0 & 0.07 & 1.200 & 3.807 \\
\hline
\end{tabular}

\begin{tabular}{|c|c|c|c|c|c|}
\hline \multicolumn{6}{|c|}{ Fancoils } \\
\hline Modelo & $\begin{array}{l}P_{\text {ref }} \\
(W)\end{array}$ & $\begin{array}{l}P_{\text {cal }} \\
(W)\end{array}$ & $\begin{array}{l}\text { Q } \begin{array}{l}\text { ref } \\
(1 / s)\end{array} \\
\end{array}$ & $\begin{array}{l}\triangle \mathrm{P}_{\text {ref }} \\
(\mathrm{kPa})\end{array}$ & $\begin{array}{l}\mathrm{PP}_{\text {ref }} \\
(\mathrm{kPa})\end{array}$ \\
\hline RFR 1 MV (A6-Planta baja) & 870.0 & 1060.0 & 0.04 & 10.800 & 11.928 \\
\hline RFR 1 MV (A7-Planta baja) & 870.0 & 1060.0 & 0.04 & 10.800 & 12.189 \\
\hline RFR 1 MV (A8-Planta baja) & 870.0 & 1060.0 & 0.04 & 10.800 & 6.817 \\
\hline RFR 1 MV (A9-Planta baja) & 870.0 & 1060.0 & 0.04 & 10.800 & 6.893 \\
\hline RFR 4 MV (A10-Planta baja) & 2800.0 & 3230.0 & 0.13 & 20.000 & 6.104 \\
\hline RFR 4 MV (A11-Planta baja) & 2800.0 & 3230.0 & 0.13 & 20.000 & 7.292 \\
\hline RFR 2 MV (A12-Planta baja) & 1300.0 & 1510.0 & 0.06 & 19.900 & 8.798 \\
\hline RFR 1 MV (A13-Planta baja) & 870.0 & 1060.0 & 0.04 & 10.800 & 4.084 \\
\hline \\
\hline $\mathrm{P}_{\mathrm{ref}}$ Potencia frigorifica total calculada & \multicolumn{5}{|c|}{ 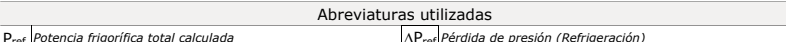 } \\
\hline$P_{\text {cal }}$ Potencia calorifica total calculada & \multirow{2}{*}{$\begin{array}{l}\Delta \text { Pref } \\
\text { PPref }\end{array}$} & rdida de pres & nacumul: & Refrigeración & \\
\hline Qref Caudal de agua (Refrigeración) & & & & & \\
\hline
\end{tabular}

Fig. 19. Results for the emitting (by space).

Installation of thermal solar energy. CTE HE 4.

The 2013 HE 4 document has hardly changed, nevertheless the modification of the estimated consumption involves completely different calculation results. In our studied case the contribution of solar domestic hot water is reduced. We need two thermal solar panels of a specific model to comply with the rules of 2006 and only one in the 2013.

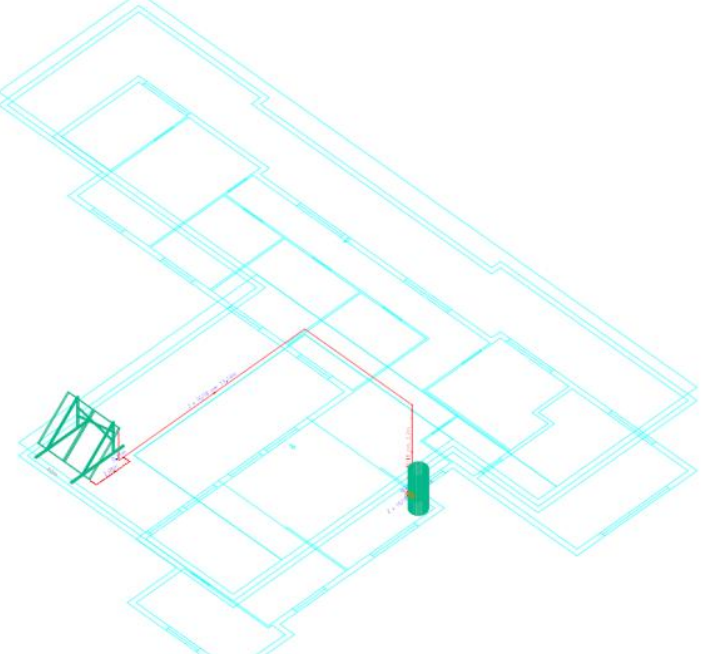

Fig. 20. Solar domestic hot water installation.

\begin{tabular}{|c|c|c|c|c|c|c|c|c|c|c|}
\hline \multicolumn{11}{|c|}{ Modelo He 2006} \\
\hline Nes & $\begin{array}{l}\text { Ocupacion } \\
{\left[\Phi_{0}\right)}\end{array}$ & $\begin{array}{c}\text { Consumo } \\
\left(\mathrm{m}^{\prime}\right)\end{array}$ & $\begin{array}{l}\text { Temperatura } \\
\text { de red } \mid \mathrm{l} C \mathrm{C}\end{array}$ & $\begin{array}{l}\text { Sollo otemico } \\
\mathrm{PCl}\end{array}$ & $\begin{array}{l}\text { Demanda } \\
\text { [Ms }\end{array}$ & $\begin{array}{l}\text { Rodocionglob } \\
\text { puimm }\end{array}$ & 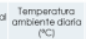 & Demancos (Mu) & $\begin{array}{l}\text { Enerdoousar } \\
\text { gimy }\end{array}$ & 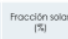 \\
\hline Enero & 100 & 79 & 9 & 36 & 1179.80 & a/s & $?$ & 1179.90 & 42.63 & $\infty$ \\
\hline Febrero & 100 & 72 & 10 & 35 & 104.73 & 1200 & 10 & $1047_{3}$ & 25329 & 76 \\
\hline & 100 & $\begin{array}{l}8.0 \\
79\end{array}$ & 11 & $\begin{array}{l}34 \\
33\end{array}$ & $\begin{array}{l}133.53 \\
1150219\end{array}$ & $\begin{array}{l}1728 \\
1100\end{array}$ & 12 & $\begin{array}{l}11323.53 \\
1025219\end{array}$ & 6807 & at \\
\hline & $\begin{array}{l}100 \\
100\end{array}$ & $\begin{array}{l}79 . \\
8.4 \\
\end{array}$ & $\begin{array}{l}13 \\
15\end{array}$ & $\begin{array}{l}{ }_{32}^{32} \\
{ }_{30}\end{array}$ & $\begin{array}{l}1052219 \\
102100\end{array}$ & $\begin{array}{l}211202 \\
2418\end{array}$ & $\begin{array}{l}14 \\
18\end{array}$ & 1052.19 & 0.000 & 101 \\
\hline $\begin{array}{l}\text { Nurio } \\
\text { fuls }\end{array}$ & 100 & 8.4 & 18 & 27 & 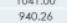 & 2028 & $\begin{array}{l}18 \\
22\end{array}$ & 90226 & 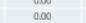 & $\begin{array}{l}107 \\
115\end{array}$ \\
\hline & 100 & 8.9 & 20 & 25 & 925.33 & 2000 & 25 & 92539 & 0.000 & 120 \\
\hline Agortio & 100 & 8.9 & 20 & 25 & 925.33 & 25.60 & 25 & 92533 & 0,000 & 118 \\
\hline Septiembere & 100 & 8.4 & 18 & 27 & 940.26 & 2020 & ${ }_{22}$ & 90228 & 0.00 & 112 \\
\hline Octubre & 100 & 8.4 & 15 & 30 & 1041.00 & 134 & 17 & $100+100$ & $\operatorname{cosec}$ & s. \\
\hline Noviemtrore & 100 & 79 & 12 & 33 & 1074.58 & 9,47 & 12 & 107.58 & .0158se & $n$ \\
\hline Divembre & 100 & 79 & 9 & 36 & 1179.80 & 7.13 & , & 1179.80 & 566.17 & 52 \\
\hline \multicolumn{11}{|c|}{ Modelo He 2013} \\
\hline Nes & $\begin{array}{l}\text { Ocupacion } \\
{[\%]}\end{array}$ & $\begin{array}{c}\text { Consumo } \\
\left(\mathrm{m}^{\prime \prime}\right)\end{array}$ & $\begin{array}{l}\text { Temperatura } \\
\text { de ted ad }\end{array}$ & $\begin{array}{l}\text { Solo ofémico } \\
\text { FCF }\end{array}$ & $\begin{array}{l}\text { Demando } \\
\text { (Mi) }\end{array}$ & 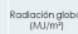 & 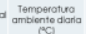 & 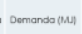 & 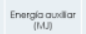 & Hosecton now \\
\hline Enero & 100 & 6.1 &, & 36 & 917.62 & Q7/5 & $?$ & 917.82 & 52000 & $\infty$ \\
\hline & 100 & & 10 & 35 & & & 10 & 8125 & 356.23 & 56 \\
\hline $\begin{array}{l}\text { Marzo } \\
\text { Aren }\end{array}$ & $\begin{array}{l}100 \\
100\end{array}$ & 6.3 & $\begin{array}{l}11 \\
13\end{array}$ & $\begin{array}{l}34 \\
33 \\
33\end{array}$ & $\begin{array}{l}{ }_{881.83}^{8.64} \\
8187\end{array}$ & $\begin{array}{l}11288 \\
21108\end{array}$ & 12 & $\begin{array}{l}831.154 \\
818937\end{array}$ & 24280 & 72 \\
\hline & $\begin{array}{l}100 \\
100\end{array}$ & $\begin{array}{ll}62 \\
6.5\end{array}$ & $\begin{array}{l}13 \\
15 \\
15\end{array}$ & $\begin{array}{l}32 \\
30\end{array}$ & $\begin{array}{l}818 . \\
809\end{array}$ & $\begin{array}{l}21102 \\
24+8\end{array}$ & $\begin{array}{l}14 \\
18 \\
18\end{array}$ & $\begin{array}{l}81837 \\
800387\end{array}$ & $\begin{array}{l}1814202 \\
113000\end{array}$ & 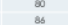 \\
\hline $\begin{array}{l}\text { Mayo } \\
\text { Junio }\end{array}$ & $\begin{array}{l}100 \\
100\end{array}$ & 6.5 & $\begin{array}{l}15 \\
18\end{array}$ & 27 & 731.31 & 2820 & $\begin{array}{l}18 \\
22\end{array}$ & 等 & 2389 & 要 \\
\hline & 100 & 69 & 20 & 25 & 719.70 & 2000 & 25 & 71900 & 0.00 & 100 \\
\hline Agosto & 100 & 6.9 & 20 & 25 & 71970 & 2563 & 25 & 719,0 & 0.000 & 101 \\
\hline Septientrere & 100 & 6.5 & 18 & 27 & 731.31 & 2020 & 22 & 731.31 & 5499 & 92 \\
\hline Octubre & 100 & 6.5 & 15 & 30 & 809.67 & 1394 & 17 & 300087 & 2205 & rs \\
\hline Noviembre & 100 & 6.1 & 12 & 33 & 835.78 & 9,47 & 12 & 835.78 & 39273 & 53 \\
\hline Dicembre & 100 & 6.1 & 9 & 36 & 917.62 & 7.13 & , & 917.02 & 576000 & 37 \\
\hline
\end{tabular}

Fig. 21. Calculation results. 


\section{CONCLUSIONS}

The study includes a final summary with lessons related to new 2013 CTE HE requirements:

The HE revision implies a greater demand reduction and zero increasing in generation through renewable. In the case we have studied, the minimum

contribution of solar heating was reduced and the contribution of electricity using photovoltaic solar did not apply.

It may be necessary to reduce thermal transmittance values by $84 \%$. That is, depending on the materials, the wall thickness can be increased to more than three times what it was.

The installation of mechanical ventilation is a determining factor in the final results.

A heat recovery system just increases the demands on the walls with respect to the previous legislation.

A facility without recovery would increase the necessary insulation values at a higher level than indicated on the first point.

The thicknesses of enclosures listed in HE appendices are only indicative and should not be used in the justification of the standars.

The default values included by calculation programs for thermal bridges are inadequate to the new requirements.

We have obtained different simulation results in different programs with the same geometry.

Reducing the demand implies the need for smaller air conditioning installations.

In our model, solar domestic hot water demand decreases.

\section{References}

[1] Directiva 2010/31/UE.

[2] Código técnico de la edificación. Documento básico de ahorro de energía. CTE DB HE.

[3] Diego Carmona Fernández, Awf Al Kassir Abdulla, Miguel Ángel Jaramillo Morán, Luis Alberto Horrillo Horrillo. Zero energy buildings rehabilitated for low energy demand by using solar and biomass energy. Current Opinion in Biotechnology ISSN: 0958-1669. Volume 24, Supplement 1, July 2013, Pages S45. 2013. Elsevier Ltd. 